\title{
Efecto de la acreditación institucional de alta calidad sobre la gestión del conocimiento
}

\author{
Carlos H. González-Campo*, Guillermo Murillo-Vargas, y Mónica García-Solarte \\ Universidad del Valle, Sede San Fernando, Cali-Colombia. (Correo-e: carlosh.gonzalez@correounivalle.edu.co; \\ guillermo.murillo@correounivalle.edu.co; monica.garcia@correounivalle.edu.co) \\ ${ }^{*}$ Autor a quien debe ser dirigida la correspondencia
}

Recibido Ago. 3, 2020; Aceptado Oct. 6, 2020; Versión final Nov. 30, 2020, Publicado Abr. 2021

\begin{abstract}
Resumen
El objetivo del presente estudio es analizar el efecto de la acreditación institucional de alta calidad, en la gestión del conocimiento en las instituciones de educación superior en Colombia. Existe evidencia del impacto de la acreditación en el desarrollo de las universidades, pero no de la incidencia de la acreditación institucional en la gestión del conocimiento. La metodología es de carácter exploratorio y utiliza métodos cuantitativos para analizar estadísticamente datos recolectados en una encuesta realizada a 272 directivos universitarios en el año 2018. Adicionalmente, se estudia el efecto en cada una de las cuatro dimensiones de la gestión de conocimiento, dada su importancia en el desarrollo de las funciones sustantivas de este tipo de instituciones. Los resultados muestran el efecto positivo y significativo de la acreditación de alta calidad en la gestión del conocimiento. Se concluye que los mejores resultados en las dimensiones de la gestión del conocimiento las poseen las instituciones acreditadas.
\end{abstract}

\section{Effect of high-quality accreditation of knowledge management in higher education institutions}

\begin{abstract}
The present study aims to assess the effect of high-quality institutional accreditation of knowledge management in higher education institutions in Colombia. There is evidence of the impact accreditation has on university development, but not for the effect of institutional accreditation on knowledge management. Quantitative methods are applied to statistically analyze data collected in a survey of 272 university managers in 2018. The effect on each of the four dimensions of knowledge management is examined, given their importance for developing essential functions. The results show that there is a positive and significant effect of high-quality accreditation on knowledge management in higher education institutions. It is concluded that accredited institutions possess the best results in knowledge management.
\end{abstract}




\section{INTRODUCCIÓN}

La mayoría de los países cuentan en educación superior, con agencias u otros organismos encargados de liderar los procesos de acreditación. En el caso colombiano, el Sistema Nacional de Acreditación fue creado hace más de 25 años, y es el Consejo Nacional de Acreditación (CNA), el encargado de liderar los procesos de acreditación de calidad, tanto de programas de formación a nivel de pregrado y postgrado, como de instituciones, es un proceso voluntario que permite altos niveles de calidad de la educación, convirtiéndose en un reconocimiento público. El concepto de alta calidad determina la orientación del proceso de acreditación Los factores evaluados en los procesos de acreditación como la productividad marcada por la investigación científica, la gestión en los procesos de internacionalización, los niveles de formación avanzada del cuerpo de profesores, las condiciones de infraestructura, los resultados académicos de sus estudiantes, el impacto en el medio social, cultural, económico de sus egresados, de manera más reciente en la política pública sobre el aseguramiento de la calidad, los resultados de aprendizaje, entre otros, han sido los elementos diferenciadores del modelo de acreditación colombiano que lo han convertido en referente internacional, no solo por ser el más antiguo de Suramérica, sino por su solidez e integralidad (CESU, 2020).

La acreditación de una institución de educación superior implica evidenciar altos niveles de calidad, en el ejercicio de sus funciones misionales. En este sentido, es estratégico que sus directivos implementen modelos de gestión del conocimiento que faciliten los procesos de acreditación y propendan por una cultura de autoevaluación y mejoramiento. Para Rodríguez y Pedraja (2016) el papel de la gestión del conocimiento liderada por las directivas es, además de un determinante estructural de la toma de decisiones, un factor que influye en la capacidad de las universidades para lograr altos estándares de calidad. Según lo planteado por Polaino et al. (2020), la enseñanza por competencias, la formación científica y la gestión del aprendizaje o gestión del conocimiento son determinantes para alcanzar la calidad y, por ende, posibilitan en mayor medida futuras acreditaciones. Además, en este análisis, encontraron que el modelo educativo-pedagógico integrado contribuye al desarrollo de importantes transformaciones cualitativas y a niveles superiores de calidad y desarrollo en la universidad estudiada.

Así mismo, en un estudio realizado en cuatro países de habla hispana (España, Chile, México y Uruguay) sobre las exigencias en los procesos de acreditación, se concluye que los estudiantes deben formarse desde currículos que incluyan temas como: la movilidad de recursos humanos, la gestión de la innovación y la gestión del conocimiento (Conchado et al., 2020). En este contexto, para Hernández-Chacón (2017), la gestión del conocimiento es importante en el desarrollo de las IES, y debe estar alineada con la misión institucional orientada a la formación de personas. De acuerdo con Abreu-Valdivia et al., (2020) también es necesario evaluar constantemente cuáles son las competencias requeridas en los docentes según la relación enseñanza-aprendizaje, por lo que identifican 40 indicadores que miden las competencias necesarias por cada sub-proceso que compone el proceso general de enseñanza-aprendizaje, entre los que se encuentra la articulación de la investigación con la formación, proceso que se evidencia en la gestión del conocimiento, desde su transferencia y posterior aplicabilidad.

Otros autores como Alhamad y Aladwan (2019), plantean la relación entre el tipo de sistema de gestión centralizado o descentralizado en las universidades, y sus sistemas internos de aseguramiento de la calidad. Anafinova (2020), estudia el proceso de descentralización en algunos países de Asia donde la regulación era una responsabilidad del gobierno. Actualmente, es el mercado desde donde se acredita y se regula a las instituciones. Según los resultados de la investigación, para las instituciones, la descentralización les ha permitido enfocarse en los procesos investigación, logrando resultados evidentes en los rankings internacionales de investigación universitaria.

Para Ahmad y Qahmash (2020), los factores críticos de éxito les permiten a las IES, comprender cómo y qué recursos se deben gestionar para el aseguramiento sostenido de la calidad académica, en especial, en acreditación como las de ABET, entre los factores identificados por los autores, se encuentra una orientación a la gestión de documentos y una cultura para el intercambio de conocimiento. Desde lo anterior, es posible plantear que la gestión del conocimiento hace parte de los elementos claves que inciden en la obtención de las acreditaciones y las certificaciones en las IES y por ende ayudan con el aseguramiento de la calidad institucional y de programas.

Haloho et al. (2018) estudia la creación de ventajas competitivas en las universidades de Indonesia, y cómo desde la administración de los recursos pueden mejorar su desempeño, demostrando que la gestión del conocimiento tiene un efecto positivo y significativo en la competitividad de las universidades. Así mismo, para Almuhaideb y Saeed (2020) existe un interés creciente desde las IES y los organismos de acreditación por la educación basada en resultados. Por su parte, El-Morsy et al. (2014), muestran el proceso de implementación de normas ISO 9001, en una universidad y su efecto en el modelo de gestión con alcance a todas las partes interesadas facilitando la cooperación interna en las universidades. 
De acuerdo con los estudios revisados, son evidentes los que han abordado el tema de la gestión y la acreditación de universidades, en algunos casos, se estudia el impacto de la acreditación en las instituciones y en los programas académicos en Colombia (Malagón et al., 2019); otros realizan análisis críticos sobre los procesos de acreditación (Delgado y González, 2018); o estudian la evolución de las políticas de aseguramiento de la calidad en instituciones de educación superior (Lima y Rubali, 2016), o finalmente, estudios de los procesos de trasformación social que con llevan los procesos de aseguramiento de la calidad en las universidades y la comparación de los modelos existentes en Chile (Díez, 2019). En esta revisión de literatura, no hay evidencia del abordaje desde la perspectiva del directivo universitario de la relación entre la acreditación institucional y la gestión del conocimiento, de ahí se desprende el objetivo de este artículo que es identificar la incidencia que tiene la acreditación institucional en la gestión del conocimiento desde la perspectiva de los directivos de las IES en Colombia.

Con relación a la gestión del conocimiento Castaneda et al. (2018), plantean que existe una relación entre el aprovechamiento del conocimiento como recurso y como mecanismo de absorción de este por medio del aprendizaje organizacional, encontrando que la transferencia del conocimiento es transversal en el aprendizaje organizacional. Esta absorción en la transferencia tiene procesos previos de creación o adquisición de conocimiento en estas organizaciones. En un ambiente científico o universitario, la gestión del conocimiento se define como la proyección, dirección, seguimiento y evaluación de acciones y decisiones para solucionar diferentes problemas o situaciones derivadas del aprendizaje, la enseñanza, la investigación y la extensión, actividades relacionadas respectivamente, con los procesos de adquisición, transmisión, creación o aplicación del conocimiento de las instituciones o sus miembros; en los que participan diferentes actores que desarrollan diversas estrategias de acción (Silvio, 1996).

El modelo y el instrumento que se utilizada en la investigación fue el desarrollado por Gomezelj et al.(2011), adaptados de los estudios de Pawlowsky en el año 2004, con el fin de aplicarlo en IES. El modelo plantea cuatro dimensiones: almacenamiento comprende la búsqueda y obtención de conocimiento de forma rápida y correcta; aplicación está ligada a la interiorización de los conocimientos adquiridos dentro de la organización, por parte de los miembros que la componen; creación o generación de conocimiento busca y crea nuevo conocimiento, nutriendo las teorías existentes o identificando nuevas tendencias antes no exploradas, que marcan una nueva en el desarrollo teórico; y la transferencia del conocimiento hace referencia la divulgación de este dentro de toda la organización y que de esta forma sea aprovechada y se desarrolle.

Para el caso de los procesos de acreditación en Colombia, el modelo desarrollado e implementado por el Consejo Nacional de Acreditación, utiliza diversos factores y características asociados con la generación, almacenamiento, transferencia de conocimiento y un poco menos con la aplicación de este. El énfasis de la evaluación de la acreditación en términos de la gestión del conocimiento, ha estado orientado a la producción científica a través de los grupos, proyectos y redes de investigación nacionales e internacionales, a los productos de investigación, libros, artículos científicos ,patentes y otros aspectos relacionados con la formación académica de los investigadores y su clasificación (Senior, Asociados o Junior), según sea su producción científica y trayectoria como investigador (CESU, 2020).

\section{METODOLOGÍA}

Este estudio busca indagar la incidencia de la acreditación de alta calidad de la institución en la gestión del conocimiento y en sus dimensiones. Los datos de esta investigación provienen de la encuesta realizada por el Grupo de Investigación Humanismo y Gestión de la Universidad del Valle, sobre las competencias directivas y su relación con la cultura organizacional, la gestión del conocimiento y los estilos de liderazgo en el contexto de 74 universidades del país y 272 directivos universitarios. El análisis de los datos fue realizado con métodos cuantitativos a través de regresiones lineales que se desarrollan en SPSS, con el objetivo de confirmar si las instituciones que son acreditadas en alta calidad inciden de manera positiva en la gestión del conocimiento de estas. La técnica utilizada en la recolección de datos fue un cuestionario que se aplicó a una muestra aleatoria no probabilística de 272 directivos universitarios de diferentes instituciones de educación superior colombianas. Para las preguntas que miden la gestión del conocimiento se utiliza una escala Likert de 30 ítems, con las opciones de respuesta entre 1 a 5 , donde 1 es total desacuerdo y 5 es total acuerdo, y para la pregunta de alta acreditación se utiliza una variable dicotómica 1 es si tiene alta acreditación institucional, y 0 no tiene acreditación institucional.

Así mismo, para evaluar la fiabilidad del índice de Gestión de Conocimiento y sus respectivas dimensiones, se realiza un análisis de fiabilidad con alfa de Cronbach. Generación de conocimiento (10 ítems; $\alpha=0,883)$, Almacenamiento de Conocimiento ( 6 ítems; $\alpha=0,852)$, Transferencia de conocimiento ( 7 ítems, $\alpha=0,903$ ) y Aplicación de conocimiento (7 ítems, $\alpha=903$ ). En la Tabla 1, se presentan los ítems medidos y sus cargas factoriales. 
Tabla 1. Factores, ítems y sus cargas factoriales.

\begin{tabular}{|c|c|c|}
\hline Factor & Ítems & $\begin{array}{l}\text { Cargas } \\
\text { Factoriales }\end{array}$ \\
\hline \multirow{11}{*}{$\begin{array}{l}\text { Genera } \\
\text { Conocimiento }\end{array}$} & $\begin{array}{l}\text { Mi Universidad soporta activamente la cooperación con otras organizaciones a través de } \\
\text { proyectos conjuntos. }\end{array}$ & 0.692 \\
\hline & $\begin{array}{l}\text { Mi Universidad se compara y toma como referente a los mejores Institutos de Educación } \\
\text { Superior en el campo. }\end{array}$ & 0.671 \\
\hline & Mi Universidad regularmente incluye practicantes calificados en los procesos educativos. & 0.629 \\
\hline & Mi Universidad tiene bien desarrolladas las actividades de investigación. & 0.750 \\
\hline & $\begin{array}{l}\text { Mi Universidad tiene bien desarrollados convenios de cooperación con otras } \\
\text { organizaciones y universidades en Investigación y Desarrollo. }\end{array}$ & 0.768 \\
\hline & $\begin{array}{l}\text { Mi Universidad alienta a sus estudiantes a participar y hacer parte de las actividades de } \\
\text { investigación. }\end{array}$ & 0.777 \\
\hline & $\begin{array}{l}\text { Mi Universidad alienta la creación de centros de Investigación y Desarrollo por los } \\
\text { empleados. }\end{array}$ & 0.618 \\
\hline & Mi Universidad alienta y soporta a los empleados en su educación avanzada. & 0.662 \\
\hline & $\begin{array}{l}\text { Mi Universidad alienta a la planta de profesores a participar y trabajar en contextos } \\
\text { internacionales. }\end{array}$ & 0.758 \\
\hline & $\begin{array}{l}\text { Mi Universidad invita a académicos conocidos para ponencias, conferencias, profesores } \\
\text { visitantes, entre otros. }\end{array}$ & 0.713 \\
\hline & Alfa de Cronbach & 0.883 \\
\hline \multirow{7}{*}{$\begin{array}{l}\text { Almacena } \\
\text { Conocimiento }\end{array}$} & $\begin{array}{l}\text { Mi Universidad regularmente almacena conocimiento (manejo de archivos) de contenidos } \\
\text { e implementación de procesos educativos }\end{array}$ & 0.809 \\
\hline & $\begin{array}{l}\text { Mi Universidad regularmente almacena conocimiento (manejo de archivos) de contenidos } \\
\text { e implementación de proyectos de investigación. }\end{array}$ & 0.808 \\
\hline & $\begin{array}{l}\text { Mi Universidad tiene una buena estructura de documentación de las competencias y } \\
\text { logros de los empleados. }\end{array}$ & 0.796 \\
\hline & Mi Universidad siempre entrevista investigadores al término de grandes proyectos. & 0.743 \\
\hline & $\begin{array}{l}\text { Mi Universidad tiene un archivo de las conferencias e investigaciones más importantes, } \\
\text { cómo ejemplo de buenas prácticas. }\end{array}$ & 0.740 \\
\hline & Mi Universidad tiene identidad organizacional bien desarrollada y conocida. & 0.699 \\
\hline & Alfa de Cronbach & 0.852 \\
\hline \multirow{8}{*}{$\begin{array}{l}\text { Transferencia } \\
\text { Conocimiento }\end{array}$} & $\begin{array}{l}\text { Mi Universidad tiene un sistema eficiente de acompañamiento y asesoría a jóvenes } \\
\text { académicos. }\end{array}$ & 0.814 \\
\hline & $\begin{array}{l}\text { Mi Universidad habilita a jóvenes académicos a tomar consciencia de diferentes temas } \\
\text { de investigación. }\end{array}$ & 0.869 \\
\hline & $\begin{array}{l}\text { Mi Universidad soporta activamente la participación en grupos de investigación } \\
\text { multidisciplinarios e interdisciplinarios. }\end{array}$ & 0.813 \\
\hline & $\begin{array}{l}\text { Mi Universidad alienta el debate en los principales conceptos y terminología de los } \\
\text { campos educativos y de investigación. }\end{array}$ & 0.843 \\
\hline & $\begin{array}{l}\text { Mi Universidad organiza regularmente presentaciones y debates sobre los logros } \\
\text { investigativos de los empleados. }\end{array}$ & 0.781 \\
\hline & Mi Universidad organiza talleres en métodos y enfoques educativos. & 0.763 \\
\hline & $\begin{array}{l}\text { Mi Universidad tiene un sistema informático eficiente que soporta la colaboración entre } \\
\text { los empleados. }\end{array}$ & 0.712 \\
\hline & Alfa de Cronbach & 0.903 \\
\hline \multirow{8}{*}{$\begin{array}{l}\text { Aplicación } \\
\text { Conocimiento }\end{array}$} & Mi Universidad aplica exitosamente las mejores prácticas en los procesos educativos. & 0.728 \\
\hline & Mi Universidad aplica exitosamente las mejores prácticas en proyectos de investigación. & 0.775 \\
\hline & Mi Universidad aplica su propia experiencia para afrontar nuevos retos. & 0.798 \\
\hline & $\begin{array}{l}\text { Mi Universidad aplica exitosamente el conocimiento disponible para el desarrollo de } \\
\text { nuevos planes de estudio. }\end{array}$ & 0.812 \\
\hline & $\begin{array}{l}\text { Mi Universidad aplica exitosamente el conocimiento disponible para el desarrollo de } \\
\text { nuevos proyectos de investigación. }\end{array}$ & 0.869 \\
\hline & Mi Universidad hace uso exitoso del potencial intelectual disponible. & 0.860 \\
\hline & $\begin{array}{l}\text { Mi Universidad aplica exitosamente el conocimiento disponible para el marketing de las } \\
\text { investigaciones y potencial educativo. }\end{array}$ & 0.750 \\
\hline & Alfa de Cronbach & 0.903 \\
\hline
\end{tabular}




\section{Análisis Estadísticos descriptivos}

Al realizar los estadísticos descriptivos de la variable gestión del conocimiento que es el promedio de la sumatoria de los 30 ítems que la miden, se identifica que el valor dado en las IES es de 4,129. En la Tabla 2, se presentan los resultados descriptivos de las dimensiones en las que se mide la gestión del conocimiento como son la generación del conocimiento $(4,279)$ que obtuvo el mayor valor promedio, seguido de la aplicación de conocimiento $(4,1448)$, luego se encuentra el almacenamiento de conocimiento $(4,055)$, por último, la transferencia de conocimiento $(4,039)$.

Tabla 2. Estadisticos descriptivos de las dimensiones de gestión del conocimiento.

\begin{tabular}{|l|c|}
\hline Índice de Gestión del Conocimiento & Media \\
\hline Generación de Conocimiento & 4,279 \\
\hline Almacenamiento de Conocimiento & 4,055 \\
\hline Transferencia de Conocimiento & 4,039 \\
\hline Aplicación de Conocimiento & 4,1448 \\
\hline
\end{tabular}

Cuando se analiza el promedio de acuerdo con la acreditación institucional, encontramos que el promedio dado a la gestión del conocimiento en las universidades acreditadas es de 4,221 y es mayor al de las universidades no acreditadas que es de 3,942. Ahora bien, en la Tabla 3 se presenta el promedio de cada uno de estos índices considerando la acreditación institucional. Con respecto a las dimensiones se puede afirmar que el promedio para la generación, almacenamiento, transferencia y aplicación de conocimiento es mayor para las instituciones acreditadas.

Tabla 3. Estadisticos descriptivos de las dimensiones de gestión de conocimiento de acuerdo a la acreditación de las Universidades

\begin{tabular}{|l|c|c|}
\hline \multirow{2}{*}{\multicolumn{1}{|c|}{ Índice de Gestión del Conocimiento }} & \multicolumn{2}{c|}{ Media } \\
\cline { 2 - 3 } & Acreditada & No Acreditada \\
\hline Generación de Conocimiento & 4,375 & 4,082 \\
\hline Almacenamiento de Conocimiento & 4,160 & 3,839 \\
\hline Transferencia de Conocimiento & 4,157 & 3,796 \\
\hline Aplicación de Conocimiento & 4,190 & 4,051 \\
\hline
\end{tabular}

Para verificar que los comportamientos univariantes diferenciales se mantienen en el análisis multivariante se incorporan variables de control, dentro del análisis de este estudio se tienen la cultura organizacional y el liderazgo, estas se definen de acuerdo a Landazury-Villalba, Jaafar-Orfale, Cristofan y Canales-Cuba (2018) quienes plantean que estos dos factores son facilitadores del flujo de conocimiento y que los directivos o gerentes de las instituciones deben tenerlos en cuenta en el momento de desarrollar procesos de transformación y de gestión, entre ellos, la gestión del conocimiento. La cultura organizacional se mide con el modelo desarrollado por Cameron y Quinn (2006), en una escala Likert (1-5), y se obtiene como media 3,867586. El liderazgo organizacional se mide con el modelo de Bass y Bolio (1994) en una escala Likert (15), y se obtiene como media 3,890406. Se plantea un modelo de regresión lineal que se estima por el método de mínimos cuadrados ordinarios, para determinar la incidencia que tienen los procesos de acreditación de alta calidad en la gestión del conocimiento. En la ecuación 1 se representa el modelo.

Gestión Conocimiento ${ }_{i}=\beta_{0}+\beta_{1}$ Acreditación $_{i}+\varepsilon_{i}$

Donde gestión conocimiento corresponde al índice de gestión del conocimiento elaborado para el directivo $i$, $\beta$ son los parámetros del modelo, $\varepsilon_{i}$ es el término de error aleatorio del modelo. Los resultados de esta estimación se presentan en la Tabla 4 . Al encontrar que se tiene una incidencia positiva de la acreditación en la gestión del conocimiento, lo que significa que cuando la universidad es acreditada se tiene una mayor gestión del conocimiento, se busca determinar si esto se evidencia de la misma manera en cada una de las dimensiones de la gestión del conocimiento como son: la generación, el almacenamiento, la transferencia y la aplicación. Por lo tanto, se plantea un modelo de regresión lineal que se estiman por el método de mínimos cuadrados ordinarios, para cada uno de los cuatro índices de gestión de conocimiento. Los mismos se representan las ecuaciones 2, 3, 4 y 5 .

Genera Conocimiento ${ }_{\mathrm{i}}=\beta_{0}+\beta_{1}$ Acreditación $+\varepsilon_{\mathrm{i}}$

Almacena Conocimiento ${ }_{i}=\beta_{0}+\beta_{1}$ Acreditación $+\varepsilon_{i}$

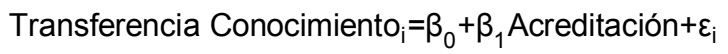

Aplicación Conocimiento $o_{i}=\beta_{0}+\beta_{1}$ Acreditación $+\varepsilon_{i}$ 
Donde gestión conocimiento corresponde con alguno de los cuatro índices gestión del conocimiento elaborados para el directivo i (generación, almacenamiento, transferencia y aplicación), $\beta$ son los parámetros del modelo, $\varepsilon_{i}$ es el término de error aleatorio del modelo. Los resultados de esta estimación se presentan en la Tabla 5.

En una segunda etapa de análisis, para verificar que los comportamientos univariantes diferenciales se mantienen en el análisis multivariante al incorporar variables de control, se consideran los índices de cultura y liderazgo organizacional. Primero con la variable general de gestión del conocimiento, teniendo en cuenta lo anterior, se propone el siguiente modelo de regresión lineal multivariante que se estiman por el método de mínimos cuadrados ordinarios.

$$
\text { Gestión Conocimiento }{ }_{i}=\beta_{0}+\beta_{1} \text { Acreditación }_{i}+\beta_{2} \text { CulturaOrganizacional }_{i}+\beta_{3} \text { Liderazgo }_{i}+\varepsilon_{i}
$$

Posteriormente se realiza en análisis multivariante en cada una de las dimensiones de la gestión del conocimiento. Teniendo en cuenta esto, se proponen los siguientes modelos de regresión lineal multivariante que se estiman por el método de mínimos cuadrados ordinarios.

$$
\begin{aligned}
& \text { Genera Conocimiento }_{i}=\beta_{0}+\beta_{1}{\text { Acreditación }+\beta_{2} \text { CulturaOrganizacional }}_{i}+\beta_{3} \text { Liderazgo }_{i}+\varepsilon_{i} \\
& \text { Almacena Conocimiento }{ }_{i}=\beta_{0}+\beta_{1}{\text { Acreditación }+\beta_{2} \text { CulturaOrganizacional }}_{i}+\beta_{3} \text { Liderazgo }_{i}+\varepsilon_{i} \\
& \text { Transferencia Conocimiento }_{i}=\beta_{0}+\beta_{1} \text { Acreditación }_{i}+\beta_{2} \text { CulturaOrganizacional }_{i}+\beta_{3} \text { Liderazgo }_{i}+\varepsilon_{i} \\
& \text { Aplicación Conocimiento }_{i}=\beta_{0}+\beta_{1}{\text { Acreditación }+\beta_{2} \text { CulturaOrganizacional }}_{i}+\beta_{3} \text { Liderazgo }_{i}+\varepsilon_{i}
\end{aligned}
$$

Donde la variable gestión conocimiento corresponde con alguno de los cuatro índices gestión del conocimiento elaborados para el directivo i (generación, almacenamiento, transferencia y aplicación), cultura organizacionali y liderazgoi corresponden a los índices de cultura organizacional y de liderazgo para el directivo $i$ respectivamente, $\beta$ son los parámetros del modelo, $\varepsilon_{i}$ es el término de error aleatorio del modelo. Los resultados de esta estimación se presentan en la Tabla 6 y 7.

\section{RESULTADOS}

En la Tabla 4 se presentan los resultados de la estimación de la ecuación 1. Estos resultados muestran que la acreditación institucional tiene un efecto positivo y significativo en la gestión del conocimiento (significancia al 1\%), es decir, que las instituciones con acreditación trabajan y se orientan a desarrollar la gestión del conocimiento.

Tabla 4. Resultados de la ecuación 1, errores estándar entre paréntesis. $\left(^{*}\right): p<0.1 ;\left(^{* *}\right): p<0.05 ;\left({ }^{* * *}\right): p<0.01$.

\begin{tabular}{|l|c|}
\hline & Gestión Conocimiento \\
\hline Acreditación Institucional & $0,2783921^{\star * *}$ \\
& $(0,0744469)$ \\
\hline Constante & $3,942121^{* *}$ \\
& $(0,0610644)$ \\
\hline $\mathrm{R}^{2}$ & 0,0492 \\
\hline
\end{tabular}

En la Tabla 5 se presentan los resultados de la estimación de las ecuaciones 2, 3, 4 y 5 respectivamente. En este caso se puede observar que, para las cuatro dimensiones de Gestión de Conocimiento, la variable Acreditación Institucional es significativa y positiva, lo cual confirma el resultado presentado en la Tabla 4 . En el caso de que la universidad se encuentre acredita existe una mayor gestión del conocimiento y también se evidencia en cada una de las dimensiones. En la generación, el almacenamiento y la trasferencia es positiva y significativa al 1\%, mientras que la aplicación de conocimiento es positivo y significativo al $10 \%$. Lo que evidencia que para las instituciones de educación superior es más complejo el llevar a la aplicación el conocimiento que se genera o que esto se evidencia cuando sus egresados están en el mundo laboral y que es difícil identificarlo desde la institución.

Tabla 5. Resultados de las ecuaciones $2,3,4$ y 5, errores estándar entre paréntesis. $\left(^{\star}\right): p<0.1 ;\left({ }^{* \star}\right): p<0.05 ;\left({ }^{\star \star \star}\right): p<0.01$

\begin{tabular}{|l|c|c|c|c|}
\hline & $\begin{array}{c}\text { Generación } \\
\text { Conocimiento }\end{array}$ & $\begin{array}{c}\text { Almacenamiento de } \\
\text { Conocimiento }\end{array}$ & $\begin{array}{c}\text { Transferencia } \\
\text { Conocimiento }\end{array}$ & $\begin{array}{c}\text { Aplicación } \\
\text { Conocimiento }\end{array}$ \\
\hline Acreditación & $0,2927195^{\star * *}$ & $0,3209758^{\star * *}$ & $0,3608911^{\star * *}$ & $0,1389817^{*}$ \\
Institucional & $(0,0746283)$ & $(0,0866442)$ & $(0,089514)$ & $(0,0827502)$ \\
\hline \multirow{2}{*}{ Constante } & $4,082022^{\star * *}$ & $3,838951^{* \star *}$ & $3,796148^{\star * *}$ & $4,051364^{* * *}$ \\
& $(0,0612132)$ & $(0,0710691)$ & $(0,073423)$ & $(0,0678751)$ \\
\hline $\mathrm{R}^{2}$ & 0,0539 & 0,0484 & 0,0568 & 0,0103 \\
\hline
\end{tabular}


Los $\mathrm{R}^{2}$ ajustados en los distintos modelos van desde 0,0103 al 0,0568. Aunque estos valores son bajos son suficientes cuando el carácter del estudio es explicativo y no predictivo, como es nuestro caso. Finalmente, en la Tabla 6 se presentan los resultados de las estimaciones de la ecuación 6 , respectivamente, donde a través del análisis multivariante al incorporar variables de control se verifican si se mantienen los comportamientos evidentes en el análisis univariante. Estos resultados revelan que la cultura organizacional tiene un impacto significativo y positivo en la gestión del conocimiento del directivo dentro de la universidad, mientras que, en el caso del liderazgo este no determina la gestión del conocimiento, y confirma que las instituciones que tienen la acreditación fomentan en mayor medida la gestión del conocimiento dentro de la institución.

Tabla 6. Resultados de la ecuación 6, errores estándar entre paréntesis. $\left({ }^{*}\right): p<0.1 ;\left({ }^{* *}\right): p<0.05 ;\left({ }^{* *}\right): p<0.01$

\begin{tabular}{|l|c|}
\hline Variable & $\begin{array}{c}\text { Gestión de } c \\
\text { onocimiento }\end{array}$ \\
\hline Acreditación & $0,31206258^{\star \star *}$ \\
Institucional & $(0,05706104)$ \\
\hline Cultura & $0,60254836^{\star \star *}$ \\
Organizacional & $(0,07025852)$ \\
\hline Liderazgo & 0,04494127 \\
& $(0,0705272)$ \\
\hline Constante & $1,4142208^{\star * *}$ \\
& $(0,24253871)$ \\
\hline R2 ajustado & 0,41073139 \\
\hline
\end{tabular}

Finalmente, en la Tabla 7 se presentan los resultados de las estimaciones de la ecuación 7, 8, 9 y 10 respectivamente. Estos resultados revelan que la cultura organizacional tiene un impacto significativo y positivo en la gestión del conocimiento del directivo dentro de la universidad, mientras que, en el caso del liderazgo este no determina la gestión del conocimiento y se confirma que la aplicación del conocimiento sigue siendo el aspecto más débil dentro del proceso.

Tabla 7. Resultados de las ecuaciones $7,8,9$ y 10, errores estándar entre paréntesis. $\left({ }^{\star}\right): p<0.1 ;\left({ }^{* *}\right): p<0.05 ;\left({ }^{* * *}\right): p<0.01$

\begin{tabular}{|c|c|c|c|c|}
\hline Variable & $\begin{array}{l}\text { Generación de } \\
\text { Conocimiento }\end{array}$ & $\begin{array}{c}\text { Almacenamiento de } \\
\text { Conocimiento }\end{array}$ & $\begin{array}{c}\text { Transferencia de } \\
\text { Conocimiento }\end{array}$ & $\begin{array}{l}\text { Aplicación de } \\
\text { Conocimiento }\end{array}$ \\
\hline $\begin{array}{l}\text { Acreditación } \\
\text { Institucional }\end{array}$ & $\begin{array}{l}0,3267348^{\star \star \star} \\
(0,06263389)\end{array}$ & $\begin{array}{c}0,35407649^{* * *} \\
(0,07283648)\end{array}$ & $\begin{array}{c}0,39648426^{* \star *} \\
(0,07293554)\end{array}$ & $\begin{array}{l}0,17095475^{*} \\
(0,06875735)\end{array}$ \\
\hline $\begin{array}{l}\text { Cultura } \\
\text { Organizacional }\end{array}$ & $\begin{array}{c}0,61361601^{* \star *} \\
(0,06498646)\end{array}$ & $\begin{array}{c}0,58731627^{\star * *} \\
(0,08108254)\end{array}$ & $\begin{array}{c}0,62931297^{\star \star \star} \\
(0,08446478)\end{array}$ & $\begin{array}{c}0,57994821^{\star * *} \\
(0,07763649)\end{array}$ \\
\hline Liderazgo & $\begin{array}{l}-0,00587452 \\
(0,06772835)\end{array}$ & $\begin{array}{c}0,09688197 \\
(0,08425765)\end{array}$ & $\begin{array}{c}0,12750752 \\
(0,08281753)\end{array}$ & $\begin{array}{c}-0,0387499 \\
(0,08013673)\end{array}$ \\
\hline Constante & $\begin{array}{l}1,7087789^{\star \star *} \\
(0,27584863)\end{array}$ & $\begin{array}{l}1,1682751^{\star \star *} \\
(0,29618383)\end{array}$ & $\begin{array}{l}0,84222286^{\star \star} \\
(0,30884217)\end{array}$ & $\begin{array}{l}1,9376065^{\star \star \star} \\
(0,29576834)\end{array}$ \\
\hline R2 ajustado & 0,40456081 & 0,3173028 & 0,35342124 & 0,26467434 \\
\hline
\end{tabular}

\section{DISCUSIÓN}

En educación superior, la mayoría de los estudios previos que indagan sobre la acreditación han estado orientados en su impacto en los programas de formación (Delgado y Gonzalez, 2018) o en la evolución de los procesos y políticas de calidad (Lima y Rubali, 2016), entre otros. La literatura muestra que la acreditación presenta una incidencia en los procesos de gestión del conocimiento en las IES, ya que, como se planeta en Conchado et al. (2020), los procesos de acreditación implican el desarrollo de distintas competencias, entre ellas, la de gestionar el conocimiento, y aunque no existen estudios previos que demuestren la relación directa y positiva entre la acreditación y la gestión del conocimiento, si hay estudios que muestran la relación de estos dos conceptos. Entre estos estudios se encuentra Polaino et al. (2020), donde se demuestra que la gestión del conocimiento es un elemento determinante para el desarrollo de la calidad. Otro de estos estudios es Rodriguez y Pedraja (2016), donde se determina que la gestión del conocimiento es un factor que influye en la capacidad de las universidades para lograr niveles de alta calidad.

En esta investigación al indagar por la incidencia que tiene la acreditación institucional en la gestión del conocimiento desde la perspectiva de los directivos de las IES en Colombia, los resultados obtenidos, tanto a nivel univariante, como multivariante (Tabla 4 y Tabla 6 , respectivamente), muestran que las instituciones que tienen alta calidad tiene un efecto positivo y significativo en la gestión del conocimiento (significancia al $1 \%$ ), es decir, que las instituciones con acreditación de alta calidad la gestión del conocimiento. Este resultado es acorde con lo expresado en Hernández-Chacón (2017) al plantear que la gestión del conocimiento es importante para el cumplimiento de las funciones misionales de las IES, así como para alcanzar estándares 
de calidad. También se puede relacionar con los resultados obtenidos en Haloho et al. (2018), cuando demuestran que la gestión del conocimiento tiene un efecto positivo y significativo en la competitividad de las universidades, debido a que los procesos de acreditación buscan que las instituciones sean más competitivas, por lo tanto, estas instituciones potencializan el desarrollo de acciones que orienten el mejoramiento de su gestión del conocimiento.

Al analizar en términos de Gomezelj et al.(2011), las cuatro dimensiones de la gestión del conocimiento, los resultados muestran (Tabla 5 y Tabla 7), que la acreditación institucional sigue teniendo una incidencia positiva y significativa en la gestión del conocimiento, siendo significativa al 1\% en la generación, almacenamiento y transferencia, mientras que es significativa al 10\% en la aplicación, lo que indica que las instituciones tienen un mayor control de los procesos de generación, almacenamiento y transferencia, pero no en el de aplicación, esto es debido a que la aplicación se evidencia en el que hacer y se relaciona cuando sus egresados están en el medio productivo, por lo tanto medirlo y controlarlo se hace más complejo para las universidades. Sin embargo, deja un reto que es importante relacionado con un seguimiento a los egresados que permita evidenciar si el conocimiento generado tiene un impacto la solución de problemas regionales, en el desarrollo territorial y en el sector productivo.

Esto también puede explicarse desde dos aspectos, el primero tendría que ver con la naturaleza de las IES, en el entendido que estas traen incorporado desde su misión institucional la generación y transferencia del conocimiento, no tanto así su aplicación. El segundo aspecto estaría más asociado al modelo propio de acreditación, tanto para los programas académicos, como para las instituciones, que ha privilegiado la producción del conocimiento, a través de la evaluación de los productos de ciencia y tecnología, como pueden ser los artículos de investigación científica y el registro de patentes de invención, entre otros y no desde la empleabilidad de sus egresados y el desarrollo regional. Por lo tanto, mediante este estudio, se ha logrado entender la incidencia de la acreditación institucional de alta calidad en la gestión del conocimiento en las IES en Colombia, debido a que impulsa su desarrollo, y, por tanto, contribuye a la materialización de la misión institucional relacionada con contribuir a la transformación de la sociedad (Arias-Coello et al., 2020).

\section{CONCLUSIONES}

A partir de los resultados obtenidos y desde la discusión propuesta en esta investigación, es posible concluir lo siguiente: 1) La acreditación institucional de alta calidad impulsa la gestión del conocimiento de las instituciones de educación superior en Colombia; 2) Los mejores resultados en las dimensiones de la gestión del conocimiento las poseen las instituciones acreditadas; 3) La cultura organizacional tiene un impacto positivo y significativo sobre la gestión del conocimiento liderada por el directivo universitario dentro de las instituciones e impulsa la gestión del conocimiento; 4) Dentro de las dimensiones de la gestión del conocimiento, la aplicación de este es la que más retos y dificultades genera a las IES en Colombia aunque la institución este acreditada.

\section{REFERENCIAS}

Abreu-Valdivia, O., Rhea-González, S., Arciniegas-Romero, G., y Guevara-López, S., Competencia para el diseño y la ejecución del proceso de enseñanza-aprendizaje en la Facultad de Ciencias Administrativas y Económicas, Universidad Técnica del Norte de Ecuador. https://doi.org/10.4067/S0718-50062020000400153, Formación Universitaria, 13(4), 153164 (2020).

Ahmad, N., y Qahmash, A., Implementing Fuzzy AHP and FUCOM to evaluate critical success factors for sustained academic quality assurance and ABET accreditation. https://doi.org/10.1371/journal.pone.0239140, Plos One, 15 (9), 1 30 (2020).

Alhamad, B.M., y Aladwan, R., Balancing centralization and decentralization management at University of Bahrain, Quality Assurance in Education, 27 (2), 237-250 (2019).

Almuhaideb, A. M., y Saeed, S., Fostering sustainable quality assurance practices in outcome-based education: lessons learned from ABET accreditation process of computing programs. https://doi.org/10.3390/su12208380, Sustainability, 12 (2), 1-26 (2020).

Anafinova, S., The role of rankings in higher education policy: coercive and normative isomorphism in Kazakhstani higher education. https://doi.org/10.1016/j.ijedudev.2020.102246, International Journal of Educational Development, 78, 1-13 (2020).

Arias-Coello, A. Simon-Martin, J. y Sanchez-Molero, J.L., Mission statements in Spanish universities. https://doi.org/10.1080/03075079.2018.1512569, Studies in Higher Education, 45:2, 299-311 (2020).

Bass, B., y Avolio, B., Improving organizational effectiveness through transformational leadership, Sage Publications, California, Estados Unidos (1994).

Cameron, K.S., y Quinn, R. E., Diagnosing and changing organizational culture, Jossey-Bass Ed., San Francisco, Estados Unidos (2006). 
Castaneda, D. I., Manrique, L. F., y Cuellar, S. Is organizational learning being absorbed by knowledge management? A systematic review. Journal of Knowledge Management, 22(2), 299-325 (2018).

CESU., Acuerdo 002 del 1 de julio de 2020. Por medio del cual se actualiza el modelo de acreditación en alta calidad, Consejo Nacional de Educación Superior Ministerio de Educación Nacional, Bogotá D.C., Colombia (2020).

Conchado Peiró, A., Carot Sierra, J. M., y Vázquez Barrachina, E., Competences of flexible professionals: validation of an Invariant Instrument across Mexico, Chile, Uruguay, and Spain. https://doi.org/doi:10.3390/su12125224, Sustainability, 12 (12), 1-13 (2020).

Díez, A.B., Structural problems of the accreditation of higher education in Chile: 2006-2012, Revista Pedagogía Universitaria y Didáctica del Derecho, 6 (1), 43-76 (2019).

Delgado, O., y González, R.E., Evaluación y acreditación desde la perspectiva de las Universidades: el Consejo de Evaluación y Acreditación Internacional, Universidades, (78), 65-74 (2018).

El-Morsy, A., Shafeek, H., Alshehri, A., y Gutub, S.A., Implementation of quality management system by utilizing ISO 9001:2008 model in the emerging faculties, Life Science Journal, 11 (8), 119-125 (2014).

Gomezelj, D., Biloslavo, R., y Trnavčevič, A., Knowledge management and organisational culture in higher education institutions content in a trusted digital archive, Rainer Hampp Verlag, 16(2), 111-139 (2011).

Haloho, E., Lumbanraja, P., Lubis, A.N., y Absah, Y., Building competitive advantage to increase organizational performance: A lesson from the private university in Medan, International Journal of Civil Engineering and Technology, 9 (12), 808-830 (2018).

Hernández-Chacón, L., Rediseño curricular de la carrera Gestión Social y Desarrollo de la Universidad de Otavalo para su proceso de acreditación. http://dx.doi.org/10.4067/S0718-50062017000600002, Formación Universitaria, 10(6), 3-16 (2017).

Landazury-Villalba, L.F., Jaafar-Orfale, H., Cristofan, M.A., y Canales-Cuba, R., Innovación y modelos de gerencia: su reflexión transformadora desde lo humano y el conocimiento, Espacios, 30(13), 1-20 (2018).

Lima, M. y Rubaii, N.M., El valor del análisis de discurso en los estudios comparativos de políticas públicas. El caso de aseguramiento de calidad en la educación superior en Colombia y Ecuador. https://doi.org/10.17533/udea.espo.n49a01, Estudios Políticos, (49),13-34 (2016).

Malagón, L.A., Rodríguez, L.H. y Machado, D.F. Políticas Públicas Educativas y aseguramiento de la calidad en la Educación Superior. https://doi.org/10.19053/01227238.4999, Revista Historia de la Educación Latinoamericana, 21(32), 273-290 (2019).

Polaino, C.J., Romillo, A. J., y Muñoz, J.F., Modelo educativo-pedagógico integrado de la Universidad de Otavalo, Ecuador. https://doi.org/10.4067/S0718-50062020000500115, Formación Universitaria, 13(5), 115-128 (2020).

Rodríguez-Ponce, E.R., y Pedraja-Rejas, L.M., Percepciones sobre la Gestión del Conocimiento de directivos universitarios de cuatro universidades Chilenas. https://doi.org/10.4067/S0718-50062016000400006, Formación Universitaria, 9(4), 41-52 (2016).

Silvio, J., Redes académicas y gestión del conocimiento en América Latina: en busca de la calidad, Educación Superior y Sociedad, 3 (2), 7-22 (1996). 
